\title{
Soil DNA pyrosequencing and fruitbody surveys reveal contrasting diversity for various fungal ecological guilds in chestnut orchards
}

Paula Baptista, ${ }^{1 \dagger}$ Francisca Reis, ${ }^{2 \dagger}$ Eric Pereira, ${ }^{1}$ Rui M. Tavares, ${ }^{2}$ Pedro M. Santos, ${ }^{3}$ Franck Richard, ${ }^{4}$ Marc-André Selosse ${ }^{5}$ and Teresa Lino-Neto ${ }^{2 *}$ ${ }^{1} \mathrm{CIMO} /$ School of Agriculture, Polytechnic Institute of Bragança, Campus de Santa Apolónia, Apartado 1172, 5301-854, Bragança, Portugal.

${ }^{2}$ BioSystems and Integrative Sciences Institute (BiolSI), Plant Functional Biology Centre, University of Minho, Campus de Gualtar, 4710-057 Braga, Portugal. ${ }^{3}$ CBMA - Centre of Molecular and Environmental Biology, University of Minho, Campus de Gualtar, 4710-057 Braga, Portugal.

${ }^{4}$ UMR 5175 CEFE, Université Montpellier - Campus CNRS, 1919 Route de Mende, 34 293, Montpellier,

France.

${ }^{5}$ Institut de Systématique, Évolution, Biodiversité (ISYEB - UMR 7205 - CNRS, MNHN, UPMC, EPHE), Muséum national d'Histoire naturelle, Sorbonne Universités, 57 rue Cuvier, CP50, 75005, Paris, France.

\section{Summary}

Fungal diversity in Mediterranean forest soils is poorly documented, particularly when considering saprobic and pathogenic organisms. Next-generation sequencing (NGS) methods applied to soil fungi provide the opportunity to unveil the most inconspicuous functional guilds (e.g. saprobes) and life forms (e.g. Corticiaceae) of this tremendous diversity. We used fruitbody surveys over 2 years and soil 454 metabarcoding in Castanea sativa orchards to evaluate respectively the reproductive (fruitbodies) and vegetative (mycelia) parts of fungal communities in three 100-year-old stands. Analysis of 839 fruitbodies and 210291 ITS1 reads revealed high fungal diversity, mainly shown by belowground analysis, with high (dominant) abundance of mycorrhizal fruitbodies and reads. Both methods displayed contrasted composition and structure of fungal communities, with Basidio- and Ascomycetes dominating above- and

Received 23 July, 2014; revised 6 September, 2015; accepted 6 September, 2015. *For correspondence. E-mail tlneto@ bio.uminho.pt; Tel. +351253601544; Fax +351253678980. 'These authors contributed equally. belowground, respectively. For the two dominant fungal guilds (i.e. ectomycorrhizal and saprobic), diversity above- and belowground overlapped weakly. This study is the first assessment of the complementarity of fruitbody surveys and NGS for analysing fungal diversity in Mediterranean ecosystems and shows that belowground methods still need to be completed by fruiting diversity to provide a comprehensive overview of the different fungal guilds. The results shed light on chestnut soil biodiversity and question the spatial distribution and synergies among fungal guilds.

\section{Introduction}

Fungi are among the most diverse organisms on Earth (Blackwell, 2011). Despite their relevance in soil and ecosystem functioning, fungal communities and their structure on a fine scale remain unknown in many ecosystems (Anderson et al., 2014; Tedersoo et al., 2014). This partly pertains to the marked discrepancy between fruiting patterns (the so-called aboveground, hereafter called the classic view) and vegetative patterns (the belowground view) of fungal communities (see Horton and Bruns, 2001, for a review) and the only recent emergence of nextgeneration sequencing (NGS) methods in fungal community ecology (Buée et al., 2009; reviewed by Orgiazzi et al., 2015). Aboveground views are based on the survey of epigeous fungal fruitbodies (Schmit and Lodge, 2005), which provide valuable information on the reproductive investment of co-occurring species but partially reflect fungal diversity. In fact, some species do not produce conspicuous fruitbodies (such as many obligatory biotrophic species) (Bidartondo and Gardes, 2005). Furthermore, fruitbody abundance represents a distorted view of the distribution of mycelia in soil (Gardes and Bruns, 1996).

Sequencing of fungal DNA, and especially internal transcribed rDNA spacer (ITS) barcoding, has allowed a renewal of the study of soil fungal communities (reviewed by Anderson and Cairney, 2004). These methods, primarily applied to mycorrhizal root tips, were recently enriched by soil-based next-generation DNA sequencing developments (Shokralla et al., 2012) to access a greater proportion of the soil fungal diversity, including saprobic 
and phytopathogenic/phytoparasitic ecological guilds (Taberlet and Coissac, 2012). However, despite the high potential for detecting huge microbial diversity, previous work has shown that issues remain when applying NGS methods to assessment of fungal diversity (Orgiazzi et al., 2015).

Previous studies compared belowground molecular approaches and aboveground fruitbody surveys, mostly limited to ectomycorrhizal (ECM) communities (e.g. Richard et al., 2005; Porter et al., 2008; Geml et al., 2009). In these comparative studies, molecular methods identified operational taxonomic units (OTUs) restricted to Agaricomycotina (Porter et al., 2008) or the Lactarius genus (Geml et al., 2009), with little overlap between above- and belowground ECM diversities. In a Quercus ilex forest, the combination of fruitbody identification and ITS barcoding on ECM root tips revealed less than $20 \%$ overlap between above- and belowground species expression (Richard et al., 2005). To our knowledge, only one study compared the ability of metabarcoding and classic methods to describe fungal diversity. This study showed a correlation between mycelial abundance and investment in reproductive structures of the fungal saprobic community inhabiting Picea abies dead wood (Ovaskainen et al., 2013). Although this reported work was not performed in soil fungal community, the results suggest that the discrepancy classically observed between above- and belowground views of ECM communities would not apply to other fungal guilds.

Sweet chestnut (Castanea sativa Mill.) is a tree species of great economic and landscaping importance in many Mediterranean countries (Castro, 2008). In recent decades, chestnut cultivation has declined dramatically in many regions because of pathogen attacks and profound societal changes (Waldboth and Oberhuber, 2009). Chestnut ecosystems are among conservation priorities in Europe (Habitat Directive 92/43/EEC, 1993) and represent a source of socioeconomic services, including mushroom picking, but paradoxically their associated fungal diversity is poorly known (for examples, see Blom et al., 2009; Baptista et al., 2010). Previous work in this ecosystem has revealed little overlap between above- and belowground fungal communities using fruitbody surveys and ITS barcoding of ECM root tips (Peintner et al., 2007), but a deeper, more detailed molecular inventory may change this picture. To the best of our knowledge, no detailed description of the fungal diversity associated with chestnut, including soil mutualistic, saprobic and parasitic guilds, has been produced so far. Here, we combine a 2-year diachronic and high-resolution fruitbody survey and ITS1 soil metabarcoding in the same chestnut orchards to provide the first comprehensive overview of the fungal diversity associated with $C$. sativa and assess the complementarity of fruitbody surveys and soil metabarcoding approaches to reveal the full diversity within Mediterranean fungal communities.

\section{Results and discussion}

Fruitbody-versus 454 belowground views of
fungal diversity

Above- and belowground fungal communities were determined from three 100-year-old non-tilled chestnut orchards from Northeast Portugal, in two nearby orchards ( $A$ and $O$ ) and another ( $\mathrm{T}$ ) located ca. $4 \mathrm{~km}$ away (Fig. $1 \mathrm{~A})$, investigating five plots per orchard. The aboveground macrofungal community, evaluated by surveys of epigeous fruitbodies from 2010 to 2011 , comprised 47 species (Table S1) belonging to 16 families and 22 genera, all from Basidiomycota. At the family level, the greatest number of species belonged to Russulaceae, Strophariaceae, Amanitaceae and Inocybaceae. At the lower taxonomic level, the most diverse genera were Russula (17\% of total number of fruiting species), Inocybe (11\%) and Amanita (11\%). In terms of abundance among the 839 harvested fruitbodies, the genera Inocybe (31\% of total number of fruitbodies), Hypholoma (28\%) and Amanita (11\%; Table S1; Fig. S1) dominated.

The 454 metabarcoding of the 30 soil cores (2 per plot, i.e. 10 per orchard) revealed a total of 210291 ITS1 raw reads (Table S2). The sequences of the 199919 high-quality fungal reads were assigned to 501 OTUs, belonging to Ascomycota (49.9\%), Basidiomycota (40.5\%), Zygomycota (5.0\%), Chytridiomycota (1.4\%) and Glomeromycota (0.6\%; Table S3). Besides taxonomically well-assigned OTUs, some OTUs were included in unclassified taxa (59 at the genus level, 72 at the family level, 20 at the order level, 31 at the class level, 13 at the phylum level). The greatest number of Ascomycota OTUs belonged to Sordariomycetes (30\% of Ascomycota), followed by Letiomycetes (19\%), whereas Basidiomycota OTUs were dominated by Agaricomycetes $(90 \%$ of Basidiomycota), $47 \%$ of which belonged to Agaricales. For Basidiomycetes, ECM species dominated in diversity and abundance: the greatest number of OTUs belonged to Thelephoraceae (14\%), Russulaceae (13\%) and Inocybaceae (7\%), and the richest genera were Russula (9\%), Inocybe (6\%), Tomentella (5\%), Cortinarius (4\%) and Amanita (3\%). In terms of abundance (considering the total number of reads, including the unclassified ones), Basidiomycota was the most abundant phylum (77\% vs. $16 \%$ for Ascomycota; Fig. S2). The most abundant Basidiomycota taxa were mainly from the Agaricomycetes class $(69 \%$ of the total number of reads), and most abundant genera were primarily ECM, first Russula (20\%) and to a lesser extent Inocybe (7\%), Tricholoma (6\%) and Cortinarius (5\%). 
$\mathbf{A}$

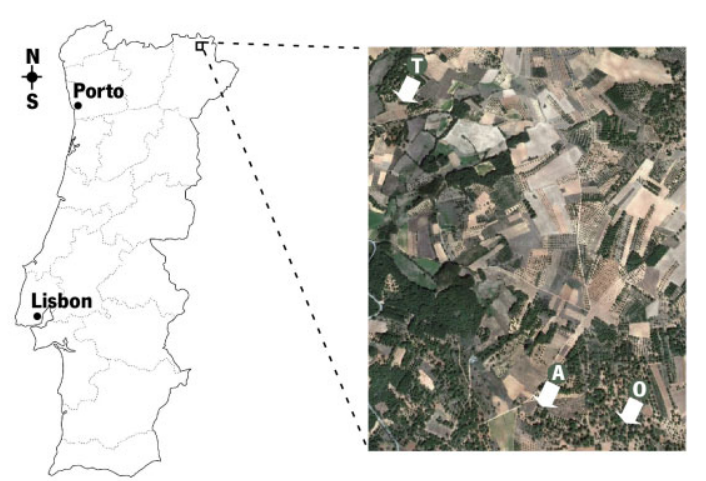

B
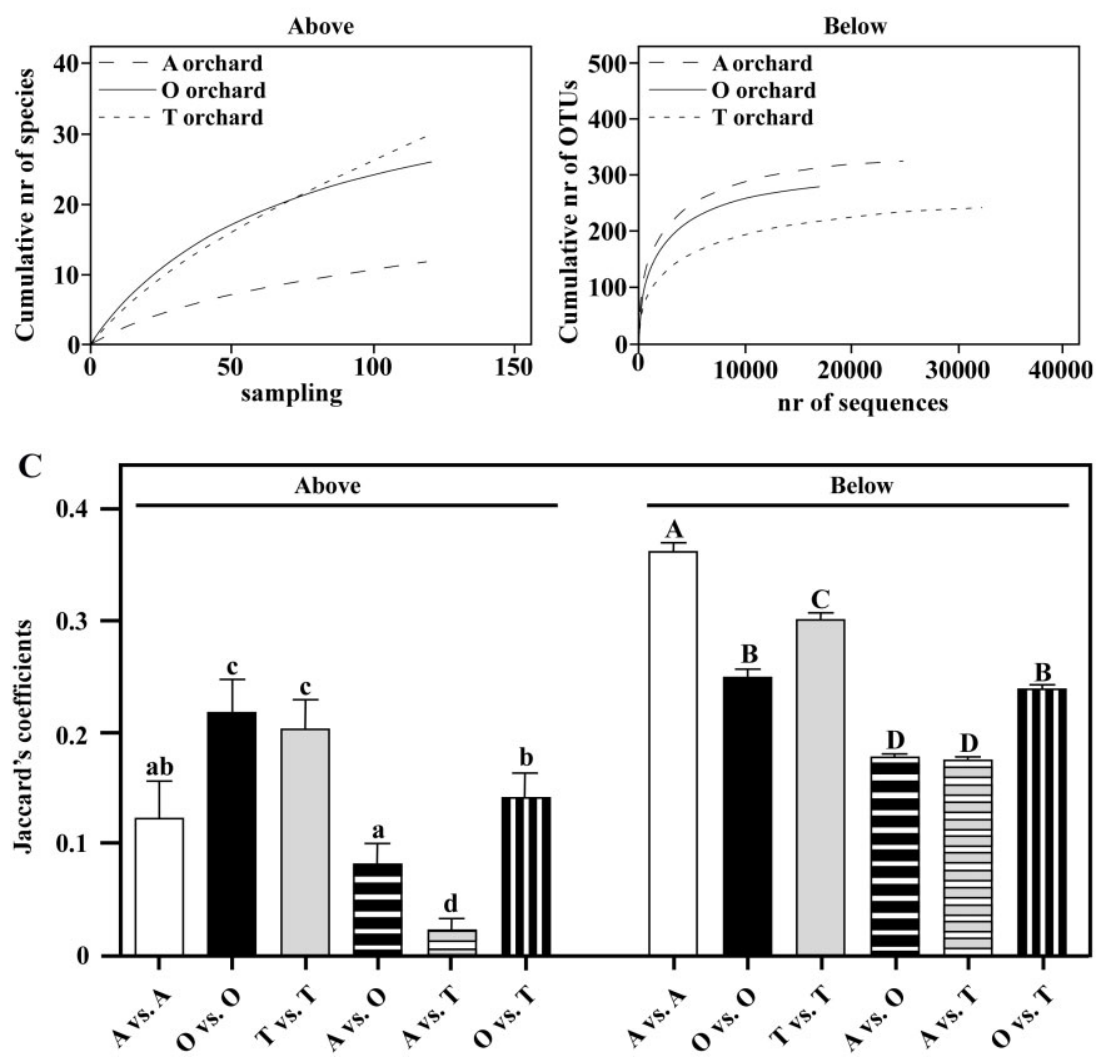

Fig. 1. Diversity of fungal communities between chestnut orchards.

A. Image created using Google Earth depicting the spatial distribution of the chestnut orchards studied. Orchards $\mathrm{A}$ and $\mathrm{O}$ (N 415 $51 \mathrm{~W} 6^{\circ} 49 ; 899 \mathrm{~m}$ altitude) were located in Oleiros, whereas orchard T was

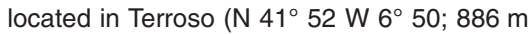
altitude), ca. $4 \mathrm{~km}$ away from $\mathrm{A}$ and $\mathrm{O}$. B. Rarefaction curves for the estimated richness of fungal communities from orchard soils ( $\mathrm{A}, \mathrm{O}$ and $\mathrm{T})$, using above- and belowground analyses. Rarefaction curves were computed by Species Diversity and Richness - version 4 discarding the singletons (Seaby and Henderson, 2006). C. Pairwise Jaccard's similarity indices between fungal communities from different plots of the same orchard (A versus $A, O$ versus $O, T$ versus $T$ ) or from plots of different orchards (A versus $O, A$ versus $T, O$ versus $T$ ). Analyses were performed for fungal communities detected by fruitbody survey ('above', on the left) or by 454 pyrosequencing of soil ('below' on the right) and were computed in the Community Analysis Package - version 4 (Henderson and Seaby, 2007). Mean values (columns) and standard deviations (bars) are reported for each comparison. Different letters indicate a significant difference at $P \leq 0.05$ (one-way ANOVA, Tukey's test). For more details, see Supporting Information.
Above- and belowground fungal communities differed markedly in composition. When considering OTUs identified at the genus level, only 13 out of 187 genera occurred in both fruitbodies and soil DNA records (Fig. 2). However, above- and belowground communities were similarly dominated in abundance and diversity by Inocybe, Amanita, Russula, and to a lesser extent by Tricholoma and Cortinarius. This result indicates that in this area of introduction, C. sativa establishes ECM associations dominated by genera that classically shape communities on other Fagaceae naturally present in the surrounding landscapes, such as Mediterranean oaks (e.g., Richard et al., 2005; Azul et al., 2010). Other well-represented genera, detected in either above- or belowground views, were not detected by the other method. The discrepancy in relative abundance detected by both approaches was more conspicuous at the species level because only five taxa (Amanita pantherina, Amanita rubescens, Bovista plumbea, Hypholoma acutum and Russula parazurea) were identified by both analyses (Fig. 2B). At the whole community level, abundances of above- and belowground species did not correlate among plots $(P=0.537$, $r^{2}=0.026$ ), but this result was expected because each approach is biased towards a particular lifestyle or reproduction form.

Our results confirm the weak correlation between fruitbodies and soil DNA distribution patterns previously reported for ECM fungal species (e.g. Kjøller, 2006; Taschen et al., 2015). This consensual discrepancy pattern has been reported for both ascomycetes, such as 


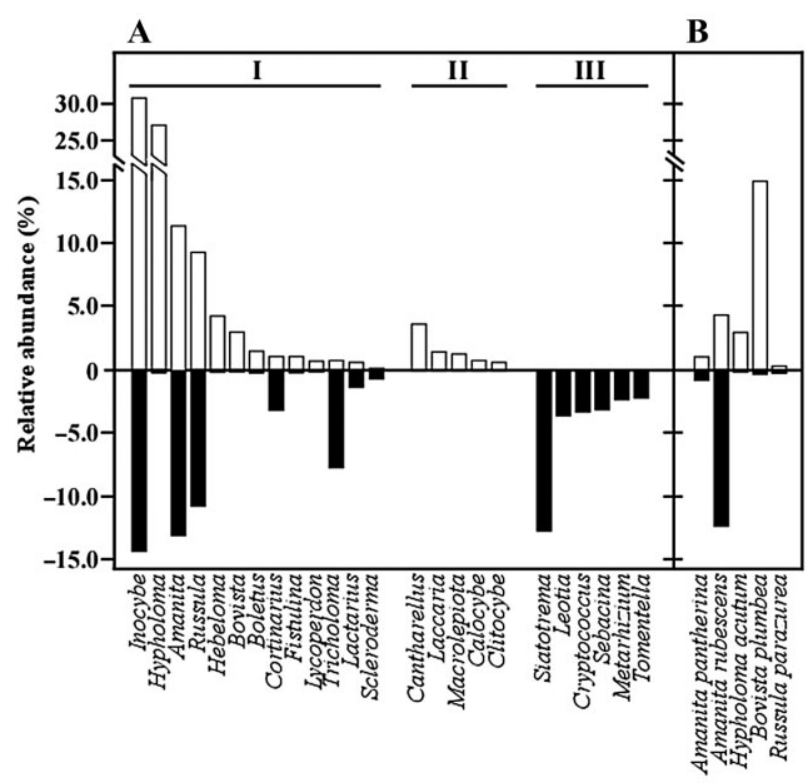

Fig. 2. Relative abundance of the most frequent fungal genera (A) found in both approaches (I) or exclusively aboveground (II) or belowground (III). The five most frequent taxa found in both approaches (B) are also illustrated. Abundance of fruitbodies and reads are, respectively, shown above or below the $\mathrm{x}$ axis. Macrofungal fruitbodies were collected within each plot weekly during the autumn (September 15 to November 31) and spring (May 1 to June 30), and biweekly in winter (December) and summer (July), in 2010-2011. On each plot, two soil cores ( $8 \mathrm{~cm}$ of diameter and $12 \mathrm{~cm}$ in depth) were collected on 13 April 2011, $2 \mathrm{~m}$ away from a single tree trunk, and the fraction between 4 and $8 \mathrm{~cm}$ in depth was analysed. After being thoroughly mixed and sieved using a $2 \mathrm{~mm}$ mesh to remove minor woody debris and roots, each of the 30 soil cores was used for DNA extraction and pyrosequencing. For each soil core, high-quality fungal sequences were assigned and classified by RDPclassifier (Wang et al., 2007). Taxonomic classification of species and OTUs was performed accordsing to Kirk and colleagues (2008). For more details, see Supporting Information.

Tuber magnatum (Zampieri et al., 2010), and Basidiomycetes, such as Hebeloma cylindrosporum, whose mycelium was not detected in soil collected more than $50 \mathrm{~cm}$ away from fruitbodies (Guidot et al., 2002). Fruitbody surveys describe the community fraction that reproduces, whereas belowground approaches reveal vegetative or even dormant (spore and sclerotia bank) individuals (Avis et al., 2006). However, belowground approaches partially compensate for the limited number of species and lack of saturation typically observed in aboveground surveys (e.g. Buée et al., 2009). The lack of correspondence between methods was suggested to be partly due to the patchiness of fungal distribution within the soil, even between close soil cores, as well as to the differential ability of fungi to occupy restricted ecological niches (e.g. rhizosphere versus soil). Similar reasons were pointed out for the low overlap between the Agaricomycetes community evaluated by fruitbodies versus soil rDNA analysis in a North American hemlock forest (Porter et al., 2008). In C. sativa forests, the identification of ECM root tips by sequencing the rDNA-ITS region and rDNA clones obtained from soil cores provided poorly overlapped views (Peintner et al., 2007). Thus, NGS and fruitbody surveys are complementary approaches to be combined in overviews of fungal communities.

\section{Spatial variation of fungal diversity patterns}

Orchards $\mathrm{O}$ and $\mathrm{T}$ presented lower total numbers of fruitbodies (192 and 123, respectively) when compared with orchard A (524), but this was due to two abundantly fruiting species $(H$. acutum and Inocybe sp. 2, which represented $84 \%$ of fruitbodies in $A$; Table $S 1$ ), so that orchard A presented fewer number of fruiting species (12) than $\mathrm{O}$ (26) and $\mathrm{T}$ (30; Fig. S3). By contrast, soils from orchard A presented more OTUs (118) than $\mathrm{O}(97)$ or $\mathrm{T}$ (98), due to differences in Ascomycetes diversity (53 OTUs in A, 37 in $\mathrm{O}$, and 34 in T).

Fungal diversity was compared among orchards by the evaluation of rarefaction curves (Fig. 1B) and computation of diversity indices (Table 1). Rarefaction curves for fruitbody surveys did not reach a plateau (Fig. S4), and confirmed that diversity was similar in $\mathrm{O}$ and $\mathrm{T}$, and lower in A. Metabarcoding rarefaction curves were closer to reaching an asymptote, suggesting a more representative

Table 1. Diversity parameters for fungal communities in orchards $\mathrm{A}, \mathrm{O}$ and $\mathrm{T}$, as seen by fruitbody survey (aboveground analysis) or 454 pyrosequencing (belowground analysis): species richness (S), Diversity rarefied to $n=24$ (Ŝ24), Simpson's index (D), Fisher's alpha, Chao1, ACE and first-order Jacknife estimates.

\begin{tabular}{lllcccccc}
\hline & Orchard & S & Ŝ24 & D & $\alpha$ Fisher & Chao1 & ACE & Jacknife \\
\hline Aboveground analysis & A & $12^{\mathrm{a}}$ & $4.47^{\mathrm{a}}$ & $2.85^{\mathrm{a}}$ & $12.65^{\mathrm{a}}$ & $12.50^{\mathrm{a}}$ & $12.02^{\mathrm{a}}$ & $12.0^{\mathrm{a}}$ \\
& $\mathrm{O}$ & $26^{\mathrm{b}}$ & $10.52^{\mathrm{b}}$ & $10.16^{\mathrm{b}}$ & $8.10^{\mathrm{b}}$ & $32.00^{\mathrm{b}}$ & $26.84^{\mathrm{b}}$ & $26.0^{\mathrm{b}}$ \\
& $\mathrm{T}$ & $30^{\mathrm{b}}$ & $9.25^{\mathrm{b}}$ & $11.22^{\mathrm{b}}$ & $2.19^{\mathrm{b}}$ & $38.64^{\mathrm{b}}$ & $32.95^{\mathrm{b}}$ & $30.0^{\mathrm{ab}}$ \\
& All & 47 & 8.89 & 6.688 & 10.77 & 96.00 & 47.8 & 47 \\
Belowground analysis & $\mathrm{A}$ & $343^{\mathrm{a}}$ & $11.46^{\mathrm{a}}$ & $31.00^{\mathrm{a}}$ & $54.28^{\mathrm{a}}$ & $351.24^{\mathrm{a}}$ & $350.81^{\mathrm{a}}$ & $343^{\mathrm{a}}$ \\
& $\mathrm{O}$ & $302^{\mathrm{b}}$ & $11.21^{\mathrm{ab}}$ & $15.89^{\mathrm{ab}}$ & $50.11^{\mathrm{b}}$ & $333.13^{\mathrm{b}}$ & $331.68^{\mathrm{b}}$ & $302^{\mathrm{b}}$ \\
& $\mathrm{T}$ & $265^{\mathrm{b}}$ & $7.03^{\mathrm{b}}$ & $19.04^{\mathrm{b}}$ & $38.19^{\mathrm{b}}$ & $292.36^{\mathrm{b}}$ & $291.05^{\mathrm{b}}$ & $265^{\mathrm{b}}$ \\
& All & 501 & 9.36 & 45.08 & 69.95 & 501.00 & 501.00 & 501 \\
\hline
\end{tabular}

In each analysis, different superscript lowercase letters denote statistically significant differences (at $P \leq 0.05$ ) between the same parameter. 
Table 2. Mantel test statistics for the correlation between community similarity or diversity and geographic distance between plots within orchards, or between orchards.

\begin{tabular}{|c|c|c|c|c|}
\hline & \multicolumn{2}{|c|}{ Aboveground } & \multicolumn{2}{|c|}{ Belowground } \\
\hline & $r(S r d)$ & $r(J c d)$ & $r($ Srd $)$ & $r(J c d)$ \\
\hline Orchard A community & -0.602 & -0.595 & -0.173 & -0.169 \\
\hline Orchard O community & -0.229 & -0.271 & -0.037 & -0.045 \\
\hline Orchard T community & 0.474 & 0.488 & 0.057 & 0.059 \\
\hline Orchard A/O community & $-0.375^{\star \star}$ & $-0.386^{\star *}$ & $-0.762^{\star \star *}$ & $-0.756^{\star \star \star}$ \\
\hline Orchard $\mathrm{A} / \mathrm{T}$ community & $-0.716^{\star * *}$ & $-0.714^{\star \star *}$ & $-0.873^{\star \star *}$ & $-0.865^{\star \star \star}$ \\
\hline Orchard $\mathrm{O} / \mathrm{T}$ community & $-0.366^{\star}$ & $-0.353^{\star}$ & $-0.383^{\star \star \star}$ & $-0.382^{\star \star \star}$ \\
\hline Whole community & $-0.283^{\star \star}$ & $-0.276^{\star \star}$ & $-0.339^{\star \star \star}$ & $-0.350^{\star \star \star}$ \\
\hline
\end{tabular}

The Mantel statistic $r(\mathrm{Srd})$ estimates the correlation between similarity (Sørensen index) and distance, and the statistic $r(\mathrm{Jcd})$ the correlation between similarity (Jaccard index) and distance. The $P$-value has been calculated using the distribution of $\mathrm{r}(\mathrm{Sd})$ estimated from 10000 permutations. ${ }^{*},{ }^{\star *}$, and ${ }^{* \star *}$ denote relationships significant at $P \leq 0.05, P \leq 0.01$ and $P \leq 0.001$, respectively.

belowground sampling. Significant differences in diversity indices for fruitbodies and OTUs were found among studied chestnut orchards (Table 1 and Table S5) and statistically supported the differences opposing orchard A, on the one side, and $\mathrm{O}$ and $\mathrm{T}$ on the other. This may be related to differences in physical or chemical factors stimulating fungal fruiting (e.g. soil moisture) as observed in other studies (reviewed by Pinna et al., 2010). The hyper-dominance of two macrofungal species (Inocybe sp.2 and H. acutum) in orchard A, only detected in the aboveground view, together with a lower fruitbody diversity, suggests that aboveground analyses are sensitive to massive (and possibly erratic) fruiting events that do not necessarily reflect belowground abundance and diversity. In fruitbody inventories, a plateau in diversity is difficult to achieve, even over a long time (e.g., Tofts and Orton, 1998), due to, at least, (i) inter-annual environmental fluctuations, triggering the fruiting of different species and (ii) community turnover, which may entail variations over years. Such limitations of fruitbody surveys are partially solved by synchronic soil metabarcoding (with the corresponding problem that the view is then not balanced over the year and seasons).

The pairwise comparison of Jaccard's coefficient between orchards, as well as between plots within orchards, revealed higher similarity based on OTUs than based on fruitbodies (Fig. 1C), although the sampling was more discontinuous belowground. For the metabarcoding, plots tended to be more similar within than between orchards. The highest Jaccard's coefficients, Sorensen's indexes and number of shared species/OTUs were recorded for orchards $O$ and $T$ (Tables S6 to S9). For fruitbody surveys, both orchards even presented slightly higher values than the plots within orchard A. By contrast, orchards $\mathrm{A}$ and $\mathrm{T}$ were the most dissimilar. Similarities between plots in terms of shared fruitbodies versus shared OTUs did not correlate for the whole fungal community (Mantel test: $r=-0.150, P=0.123$; Table S4) or even for the most represented phylum Basidiomycota $(r=-0.058, P=0.564)$. A significant correlation was only found for the most abundant Basidiomycota OTUs, comprising those with more than 10 reads in the whole study, and fruitbodies $(r=0.218, P=0.031)$.

When considering distance between sampling plots, fungal community similarity decreased as geographic distance increased (Table 2), independently of the sampling method (fruitbodies or OTUs). Within the same orchard, in which the most separated plots were $250 \mathrm{~m}$ apart, most correlations were negative and non-significant. The picture was different at higher scales, where Mantel tests revealed significant negative correlation between similarity indexes and distance between orchards, exemplifying the usually low compositional drift with distance obtained in these communities previously found using fruitbody surveys (Richard et al., 2004). When evaluating plots from two different orchards, the most negative and significant correlation was not found for the most separated plots $(\mathrm{O}$ and $\mathrm{T})$, suggesting that distance is not the sole factor driving divergence at this scale, and that other parameters (e.g., soil chemistry, history of practices, plant community features) could drive spatial heterogeneity (de Vries et al., 2012). Indeed, in contrast to plant and animal taxa, microbial distribution seems primarily controlled by edaphic variables (van der Gast et al., 2011). The significant divergence between plots emphasizes the importance of selecting the appropriate soil sampling and sample pooling strategy, which greatly influence the description of the soil microbial community (Manter et al., 2010). Here, with two independent soil replicates from each plot resulting in 10 cores per orchard, we expected to minimize variability within orchards and to characterize fungal communities better. Indeed, not only was a plateau almost reached in community description, but also Jaccard's and Sørensen's similarity coefficients were always higher between orchards for metabarcoding than for fruitbody surveys. However, because the evaluation of fruitbodies covers a wider sampling area/time and the metabarcoding is spatially and temporally restricted, the 


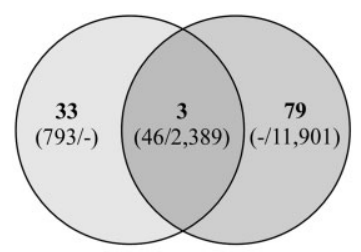

Mycorrhizal

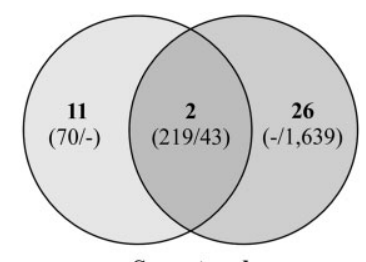

Saprotroph

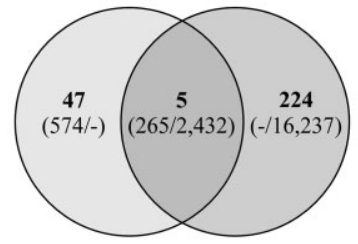

Total

Fig. 3. Number of mycorrhizal, saprobic and total species/OTUs detected by above- (dark grey), belowground (light grey) or by both approaches (overlapping area), as well as their abundance (number of fruitbodies/reads in brackets). Data correspond to all detected fruitbodies and OTUs identified up to the genus $(97 \%$ identity) that presented more than 5 reads over the 30 soil cores.

combination of above- and belowground analyses provides a more complete view of the soil fungal community (see also Halme et al., 2012); we thus recommend this dual approach.

\section{Relation between ecological guilds}

We grouped the identified species/genera (from fruitbodies and OTUs identified at least to genus level and represented by five or more reads) into guilds defined by trophic strategies (Tables S1 and S3). ECM species dominated the aboveground fungal community (in richness and abundance) with $64.5 \%$ of fruitbodies, whereas saprobic and phytoparasitic species represented $34.5 \%$ and $1 \%$ of the total, respectively. Most reads $(77.1 \%)$ and OTUs (50\%) belonged to mycorrhizal fungi (Table S3). Saprobic species were second in abundance (9\% of reads) and diversity (22.7\% of OTUs). Last, phytopathogenic/phytoparasitic organisms accounted for only $5.8 \%$ of reads and $9.3 \%$ of OTUs. Identified phytopathogens are unable to infect chestnut trees, and we found no species causing severe chestnut diseases (like Cryphonectria parasitica; our primers cannot detect Oomycota such as the ink disease agents) (Anagnostakis, 1995).

The complementarity of above- and belowground views of community composition was consistently low for the dominant ecological guilds (Fig. 3). A higher diversity was obtained by metabarcoding for all guilds, which is not surprising when considering the deeper sampling effort and the fact that the majority (estimated 58\%) of metabarcoded OTUs do not produce visible fruitbodies. But even among species able to fruit, the two methods revealed a limited overlap: only $6.7 \%$ of soil fungal

OTUs were represented by fruitbodies and, reciprocally, metabarcoding failed to detect $89 \%$ of the fruiting species. This pattern was consistent across ecological guilds (Fig. 3). Even among fruiting species, fructification is affected by habitat and climatic conditions in a complex way, so that the amount of vegetative mycelium is not its sole predictor (Peintner et al., 2007). Moreover, contrasted above- and belowground sampling designs may also contribute to the weak diversity overlap, especially for the saprobic guild: while soil cores were collected at a limited depth (from 4 to $8 \mathrm{~cm}$ ) from spatially discontinuous, limited locations, fruitbody surveys were the expression of the whole soil profile in a continuous area (plot), even if hypogeous and crustose diversity was not surveyed. Restricting metabarcoding to a focal soil horizon that did not include the surface organic soil layers may have discarded (i) saprobes that dominate in the organic horizon (e.g. Voříšková et al., 2014), including those able to form fruitbodies and (ii) some ECM species, whose mycelia can be concentrated at exclusive levels of the soil profile (e.g Anderson et al., 2014). Lastly, difference in time and duration of sampling of soil cores (on 13 April 13 2011) and fruitbodies (fruiting events from January 2010 to December 2011) could have affected the results: any temporal dynamics of fungal communities (see Richard et al., 2011; Voříšková et al., 2014) may have limited metabarcoding representativity.

In conclusion, this study is, to the best of our knowledge, the first comparison of the soil fungal community by fruitbody survey (aboveground approach) versus metabarcoding (belowground approach). Besides the detection of the mycorrhizal community, it provides a belowground view of the parasitic and saprobic fungal community and reveals previously undetected diversity in chestnut orchards. Because practical limitations still constrain fruitbody detection and soil sampling, the two approaches remain complementary in depicting the soil fungal community because they provide non-overlapping results. Fungi with clustered, patchy distribution could have slipped out of 454 pyrosequencing, whereas temporally erratic production of fruitbodies limits the efficiency of the aboveground view. Additionally, the detection of ecological guilds other than the ECM one was only possible by metabarcoding. The balance between ecological guilds in soils provides the basis for further work, exploring fungal interactions in soil and the roles of plant-fungi interactions in the functioning of chestnut orchard ecosystems.

\section{Data accessibility}

Sequence data have been deposited in the National Centre for Biotechnology Information (NCBI) Sequence Read Archive (SRA) under accession number SRP051057. 


\section{Acknowledgements}

This work was funded by FEDER through the Operational Competitiveness Program (COMPETE) and by Portuguese national funds through the Foundation for Science and Technology (FCT) within the scope of project PTDC/AGR-AAM/ 099556/2008 'Effect of the fungus Hypholoma fasciculare on the sustainability of Castanea sativa orchards'. The authors would like to thank to Biocant (especially Dr. Conceição Egas) for pyrosequencing support.

\section{References}

Anagnostakis, S.L. (1995) The pathogens and pests of chestnuts. Adv Botanical Res 21: 125-145.

Anderson, I.C., and Cairney, J.W.G. (2004) Diversity and ecology of soil fungal communities: increased understanding through the application of molecular techniques. Environ Microbiol 6: 769-779.

Anderson, I.C., Genney, D.R., and Alexander, I.J. (2014) Fine-scale diversity and distribution of ectomycorrhizal fungal mycelium in a Scots pine forest. New Phytol 201: 1423-1430.

Avis, P.G., Dickie, I.A., and Mueller, G.M. (2006) A 'dirty' business: testing the limitations of terminal restriction fragment length polymorphism (TRFLP) analysis of soil fungi. Mol Ecol 15: 873-882.

Azul, A.M., Sousa, J.P., Agerer, R., Martín, M.P., and Freitas, H. (2010) Land use practices and ectomycorrhizal fungal communities from oak woodlands dominated by Quercus suber L. considering drought scenarios. Mycorrhiza 20: 73-88.

Baptista, P., Martins, A., Tavares, R.M., and Lino-Neto, T. (2010) Diversity and fruiting pattern of macrofungi associated with chestnut (Castanea sativa) in the Trás-os-Montes region (Northeast Portugal). Fungal Ecol 3: 9-19.

Bidartondo, M., and Gardes, M. (2005) Fungal diversity in molecular terms: profiling, identification, and quantification in the environment. In The Fungal Community: Its Organization and Role in the Ecosystem. Dighton, J., White, J.F., and Oudemans, P. (eds). Boca Raton, Florida: CRC Press, pp. 215-240.

Blackwell, M. (2011) The fungi: 1, 2, 3 . . 5.1 million species? Am J Bot 98: 426-438.

Blom, J.M., Vannini, A., Vettraino, A.M., Hale, M.D., and Godbold, D.L. (2009) Ectomycorrhizal community structure in a healthy and a Phytophthora-infected chestnut (Castanea sativa Mill.) stand in central Italy. Mycorrhiza 20: 25-38.

Buée, M., Reich, M., Murat, C., Morin, E., Nilsson, R.H., Uroz, S., and Martin, F. (2009) 454 Pyrosequencing analyses of forest soils reveal an unexpectedly high fungal diversity. New Phytol 184: 449-456.

Castro, M. (2008) Silvopastoral systems in portugal: current status and future prospects. In Agroforestry in Europe. Rigueiro-Rodríguez, A., McAdam, J., and MosqueraLosada, M.R. (eds). Dordrecht, the Netherlands: Springer, pp. 111-126.

Gardes, M., and Bruns, T.D. (1996) Community structure of ectomycorrhizal fungi in a Pinus rnuricata forest: aboveand below-ground views. Can J Bot 74: 1572-1583. van der Gast, C.J., Gosling, P., Tiwari, B., and Bending, G.D. (2011) Spatial scaling of arbuscular mycorrhizal fungal diversity is affected by farming practice. Environ Microbiol 13: 241-249.

Geml, J., Laursen, G.A., Timling, I., McFarland, J.M., Booth, M.G., Lennon, N., et al. (2009) Molecular phylogenetic biodiversity assessment of arctic and boreal ectomycorrhizal Lactarius Pers. (Russulales; Basidiomycota) in Alaska, based on soil and sporocarp DNA. Mol Ecol 18: 22132227.

Guidot, A., Debaud, J., and Marmeisse, R. (2002) Spatial distribution of the below-ground mycelia of an ectomycorrhizal fungus inferred from specific quantification of its DNA in soil samples. FEMS Microbiol Ecol 42: 477486.

Halme, P., Heilmann-Clausen, J., Rämä, T., Kosonen, T., and Kunttu, P. (2012) Monitoring fungal biodiversity - towards an integrated approach. Fungal Ecol 5: 750-758.

Henderson, P.A., and Seaby, R.M.H. (2007) Community Analysis Package 4.0. Lymington, UK: Pisces Conservation.

Horton, T.R., and Bruns, T.D. (2001) The molecular revolution in ectomycorrhizal ecology: peeking into the black-box. Mol Ecol 10: 1855-1871.

Kirk, P.F., Cannon, P.F., Minter, D.W., and Stalpers, J.A. (2008) Dictionary of the Fungi, 10th edn. Wallingford, UK: CAB International.

Kjøller, R. (2006) Disproportionate abundance between ectomycorrhizal root tips and their associated mycelia. FEMS Microbiol Ecol 58: 214-224.

Manter, D.K., Weir, T.L., and Vivanco, J.M. (2010) Negative effects of sample pooling on PCR-based estimates of soil microbial richness and community structure. Appl Environ Microbiol 76: 2086-2090.

Orgiazzi, A., Dunbar, M.B., Panagos, P., de Groot, G.A., and Lemanceau, P. (2015) Soil biodiversity and DNA barcodes: opportunities and challenges. Soil Biol Biochem 80: 244250.

Ovaskainen, O., Schigel, D., Ali-Kovero, H., Auvinen, P., Paulin, L., Nordén, B., and Nordén, J. (2013) Combining high-throughput sequencing with fruit body surveys reveals contrasting life-history strategies in fungi. ISME J 7: 16961709.

Peintner, U., lotti, M., Klotz, P., Bonuso, E., and Zambonelli, A. (2007) Soil fungal communities in a Castanea sativa (chestnut) forest producing large quantities of Boletus edulis sensu lato (porcini): where is the mycelium of porcini? Environ Microbiol 9: 880-889.

Pinna, S., Gévry, M., Côté, M., and Sirois, L. (2010) Factors influencing fructification phenology of edible mushrooms in a boreal mixed forest of Eastern Canada. For Ecol Manage 260: 294-301.

Porter, T.M., Skillman, J.E., and Moncalvo, J.-M. (2008) Fruiting body and soil rDNA sampling detects complementary assemblage of Agaricomycotina (Basidiomycota, Fungi) in a hemlock-dominated forest plot in southern Ontario. Mol Ecol 17: 3037-3050.

Richard, F., Moreau, P.-A., Selosse, M.-A., and Gardes, M. (2004) Diversity and fruiting patterns of ectomycorrhizal and saprobic fungi in an old-growth Mediterranean forest dominated by Quercus ilex L. Can J Bot 82: 1711-1729. 
Richard, F., Millot, S., Gardes, M., and Selosse, M.-A. (2005) Diversity and specificity of ectomycorrhizal fungi retrieved from an old-growth Mediterranean forest dominated by Quercus ilex. New Phytol 166: 1011-1023.

Richard, F., Roy, M., Shahin, O., Sthultz, C., Duchemin, M., Joffre, R., and Selosse, M.-A. (2011) Ectomycorrhizal communities in a Mediterranean forest ecosystem dominated by Quercus ilex: seasonal dynamics and response to drought. Ann Forest Sci 68: 57-68.

Schmit, J.P., and Lodge, D.J. (2005) Classical methods and modern analysis for studying fungal diversity. In Fungal Community: Its Organization and Role in the Ecosystem. Dighton, J., White, J.F., and Oudemans, P. (eds). Boca Raton, Florida: CRC Press, pp. 193-214.

Seaby, R.M., and Henderson, P.A. (2006) Species Diversity and Richness Version 4. Lymington, UK: Pisces Conservation.

Shokralla, S., Spall, J.L., Gibson, J.F., and Hajibabaei, M. (2012) Next-generation sequencing technologies for environmental DNA research. Mol Ecol 21: 1794-1805.

Taberlet, P., and Coissac, E. (2012) Towards next-generation biodiversity assessment using DNA metabarcoding. Mol Ecol 33: 2045-2050.

Taschen, E., Sauve, M., Taudiere, A., Parlade, J., Selosse, M.-A., and Richard, F. (2015) Whose truffle is this? Distribution patterns of ectomycorrhizal fungal diversity in Tuber melanosporum brûlés developed in multi-host Mediterranean plant communities. Environ Microbiol 17: 2747-2761. doi:10.1111/1462-2920.12741.

Tedersoo, L., Bahram, M., Põlme, S., Kõljalg, U., Yorou, N.S., Wijesundera, R., et al. (2014) Global diversity and geography of soil fungi. Science 346: 1256688. doi:10.1126/ science.1256688.

Tofts, R.J., and Orton, P.D. (1998) The species accumulation curve for agarics and boleti from a Caledonian pinewood. Mycologist 12: 98-102.

Voříšková, J., Brabcová, V., Cajthaml, T., and Baldrian, P. (2014) Seasonal dynamics of fungal communities in a temperate oak forest soil. New Phytol 201: 269-278.

de Vries, F.T., Manning, P., Tallowin, J.R.B., Mortimer, S.R., Pilgrim, E.S., Harrison, K.A., et al. (2012) Abiotic drivers and plant traits explain landscape-scale patterns in soil microbial communities. Ecol Lett 15: 1230-1239.

Waldboth, B.M., and Oberhuber, W. (2009) Synergistic effect of drought and chestnut blight (Cryphonectria parasitica) on growth decline of European chestnut (Castanea sativa). For Path 39: 43-55.

Wang, Q., Garrity, G.M., Tiedje, J.M., and Cole, J.R. (2007) Naive bayesian classifier for rapid assignment of rRNA sequences into the new bacterial taxonomy. Appl Environ Microbiol 73: 5261-5267.

Zampieri, E., Murat, C., Cagnasso, M., Bonfante, P., and Mello, A. (2010) Soil analysis reveals the presence of an extended mycelial network in a Tuber magnatum truffleground. FEMS Microbiol Ecol 71: 43-49.

\section{Supporting information}

Additional Supporting Information may be found in the online version of this article at the publisher's web-site:
Fig. S1. Taxonomic distribution of all macrofungi collected. Species distribution is exhibited using Krona charts with Microsoft Excel.

Fig. S2. Taxonomic distribution of all fungal identified reads. OTUs assignment and classification were performed by RDPclassifier of filtered reads, and OTUs distribution is exhibited using Krona charts with Microsoft Excel.

Fig. S3. Venn diagrams representing shared macrofungal species (a), total number of OTUs (b) and Basidiomycota OTUs (c) between the three studied chestnut orchard sites. Each orchard is referred by $\mathrm{A}, \mathrm{O}$ or $\mathrm{T}$.

Fig. S4. Rarefaction curves for the estimated richness of microbial populations from orchard plot soils, using the aboveand belowground analyses. For aboveground analysis (a, c, e) the number of identified species is represented as a function of the sampling number, and for belowground analysis $(b, d, f)$ the number of OTUs (identified at $97 \%$ identity) is represented as a function of the number of reads, both for soil samples A (a, b), $\mathrm{O}(\mathrm{c}, \mathrm{d})$ and $\mathrm{T}(\mathrm{e}, \mathrm{f})$. Curves were generated by Species Diversity and Richness (version 6.0) software, discarding the singletons. Each site is referred by two letters: A, O or T (first letter) corresponds to the chestnut orchard, and A to $E$ (second letter) refers to each plot.

Table S1. Macrofungal species collected in each chestnut orchard $(A, O$ and $T)$ during two consecutive years. Species were classified by their ecological guild (EG) as mycorrhizal $(\mathrm{M})$, phytoparasitic (P) or saprobic (S).

Table S2. Number of reads obtained by 454 sequencing of DNA samples taken from $A, O$ and $T$ chestnut orchards. The total raw number of reads was subjected to a first quality filtering using CLC Genomics Workbench (v8). Filtered and trimmed datasets were then classified by RDPclassifier. The number of unclassified reads found in each orchard is presented, being the unclassified reads at genus level displayed in brackets.

Table S3. Identified OTUs and corresponding number of reads found in each chestnut orchard soil. Only those OTUs identified up to the genus, with more than $97 \%$ identity, and represented by five or more reads are displayed. The ecological guild (EG) of each OTU is also displayed: mycorrhizal $(\mathrm{M})$, parasitic $(\mathrm{P})$, saprobic $(\mathrm{S})$, parasitic/saprobic $(\mathrm{PS})$ or classification not available (NA).

Table S4. Occurrence of shared fungal species detected by a fruitbody survey (upper side of the table) and shared OTUs (with genus identified at $97 \%$ identity) (lower side of the table) between plots. Values correspond to relative abundance (in percentage) for each plot combination. Each plot is referred by two letters: A, O or T (first letter) corresponds to the chestnut orchard, and A to $E$ (second letter) refers to each plot.

Table S5. Diversity parameters for fungal communities from the studied orchard plots, after surveying the fungal community by fruitbody (aboveground analysis) or pyrosequencing (belowground analysis) methods: Species richness (S), Diversity rarefied to $n=24$ (Ŝ24), Simpson's index (D), Shannon index $\left(\mathrm{H}^{\prime}\right)$, alpha Fisher, Chao1, ACE, and 1st order Jacknife estimates. The highest and lowest estimates are highlighted in bold, being the highest also underlined. Each site is referred by two letters: $A, O$ or $T$ (first letter) corresponds to the chestnut orchard, and A to $E$ (second letter) refers to each plot. 
Table S6. Similarity matrixes of the number of macrofungal species shared between two sampling plots (upper side of the table) and the geographical distance (in meters) between two localities (lower side of the table). Each site is referred by two letters: A, O or T (first letter) corresponds to the chestnut orchard, and A to E (second letter) refers to each plot.

Table S7. Similarity matrixes of the number of shared OTUs between two soil cores (upper side of the table) and the geographical distance (in meters) between two localities (lower side of the table). Each site is referred by two letters: $\mathrm{A}, \mathrm{O}$ or $\mathrm{T}$ (first letter) corresponds to the chestnut orchard, and $A$ to $E$ (second letter) refers to each plot.
Table S8. Coefficients of similarity between orchard plots using an aboveground analysis. The Jaccard's coefficient is displayed in the upper side of the table and the Sørensen's coefficient in the lower side. Each site is referred by two letters: $\mathrm{A}, \mathrm{O}$ or $\mathrm{T}$ (first letter) corresponds to the chestnut orchard, and A to $E$ (second letter) refers to each plot.

Table S9. Coefficients of similarity between orchard plots using a belowground analysis. The Jaccard's coefficient is displayed in the upper side of the table and the Sørensen's in the lower side. Each plot is referred by two letters: A, O or T (first letter) corresponds to the chestnut orchard, and A to $E$ (second letter) refers to each plot. 